A - Research concept and design

B - Collection and/or assembly of data

C - Data analysis and interpretation

D - Writing the article

E - Critical revision of the article

F - Final approval of article

\section{Effect of voluntary muscle contraction on postural stability in healthy adults}

\author{
Justyna Kędziorek*B-D (D), Michalina Błażkiewicz ${ }^{A, E-F}$ (D) \\ Józef Piłsudski University of Physical Education, Warsaw, Poland
}

*Correspondence: Justyna Kędziorek; Józef Piłsudski University of Physical Education, Warsaw, Poland; email: justyna.kedziorek@awf.edu.pl

\begin{abstract}
Introduction: Postural control is a complex system that combines many subcomponents. The central nervous system's connection with the muscular system allows to execute the voluntary movements and provides appropriate tonus and contractions of postural muscles. The aim of this study was to assess the effect of voluntary muscle contraction on the stability of standing posture.

Material and methods: Seventeen young adults (24.11 \pm 3.05 years) completed 4 bipedal standing attempts lasting 20 seconds each. Measurements were performed on AMTI plate and involved maintaining balance while standing barefoot on both feet with eyes open and closed. Two additional tests with provoked isometric voluntary muscle contraction were performed under the same visual conditions.

Results: For examined healthy subjects, the lack of visual feedback in combination with increased muscle tension caused a significant increase $(\mathrm{p}<0.05)$ in the peak of sway in the anterior-posterior $(\mathrm{AP})$ and medio-lateral (ML) direction. On the other hand, the values of the center of pressure $(\mathrm{CoP})$ path length and its velocity due to the increased muscle contraction did not significantly decrease their values for trials with eyes open and closed.

Conclusions: This study indicates that voluntary muscle contraction can influence the standing posture mainly in combination with a lack of visual feedback, causing its deterioration.
\end{abstract}

Keywords: postural balance, posturography, muscle tonus

\section{Introduction}

Postural control is a complex and multifactorial mechanism $[1,2]$. It includes many components: movement and sensory strategies, orientation in space, control of dynamics, cognitive processing, and biomechanical constraints [3]. The impairment of one mentioned above component leads to postural instability and to the activation of the compensation mechanism [4]. These situations may arise during muscle injuries and neurological diseases [5]. One group of muscles has to do more work to take the strain off and compensate for weakness or ineffective contractions of other muscles group [6]. Additionally, simple factors such as time of a day [7], global and local fatigue [8], age [9], BMI and others [10] may affect postural stability. Thus, its 
assessment is challenging because a lot of external and internal components need to be evaluated to explore, which of them is responsible for possible instability [3]. To find the reason for instability, researchers manipulating different factors like the support area (bipedal or unipedal standing) [11-13], type of surface (hard, soft-foams or unstable) [14], visual conditions (with or without feedback) [11]. The convenient evaluation and verification of postural control are crucial from the physiotherapy point of view. The acquired knowledge can ensure the implementation of appropriate rehabilitation or treatment [3].

A small number of studies have focused on assessing the effect of muscle tone on postural stability. The researchers focused more on strategies and sequences during perturbed and unperturbed standing, such as Blenkinsop, Pain [15]. Others describing stages of body balance regulation, separately for anterior-posterior and medio-lateral direction [16]. There are also studies assessing the effect of muscle strengthening on postural stability after performing core exercises, Pilates or Tai-Chi [17-19]. By contrast, Ivanenko and Gurfinkel [20] in their study emphasize the meaningfulness of the investigation postural tone on postural stability. The postural tone is usually associated with antigravity support, represents the activation of muscles to afford an explicit postural attitude. The body posture in different humans is actuated both by the individual morphology and unique low muscle activity, which can be significantly affected by diseases [20]. This phenomenon is less known and much less studied [21,22]. Benjuya, Melzer [23] examined the elderly and young, and compared their muscle activity using EMG signals. The participants had trials on a narrow and wide base of support, with and without visual control. In the elderly group, the activation of muscles was larger, than in younger, regardless of the base of support. The major differences have been found in soleus and tibialis muscles. The hamstrings were also more used in the older group rather than younger. The authors suggested that it was probably due to needed hip stabilization. The conclusion was, that the elderly increased their sense of stability by maintaining the co-contractions. There were low soleus and tibialis anterior EMG ratio. They do not use cutaneous and proprioceptive information to replace vision, but the increased muscle co-contractions. Cenciarini, Loughlin [25] emphasized that increased muscle co-contraction is a compensatory balance response. It is observed in older adults. Generally, it is assumed that the elderly enhance stability by increasing joint stiffness. The results of Tassani, Font-Llagunes's work [24] based on the young group, can infer and explain, that increased muscle co-contraction in the elderly, with a potential fall risk, decreased their stability. . It is worth mentioning that this strategy leads to increasing energy costs, stress and stiffness, and it is associated with impaired postural control $[25,26]$. That phenomenon appears also in Parkinson's disease [27]. Moreover, it should be noted the increased muscular co-contraction could be related to mental stress or pathological conditions. Mental or physical stress brings inability and difficulties to relax, leads to increased muscular tension mainly of trapezius muscles, pelvic floor muscles, shoulder girdle muscles and gluteus [28]. Consequently, it affects the overload of the locomotor system and low-back pain [9]. In addition, in certain diseases (depression, general anxiety disorder, schizophrenia), where there is a high level of anxiety or stress as well as increased muscles tension, the parameters of postural control may deteriorate $[29,30]$.

Taking into account the above-mentioned reports, it is important to examine whether in young people deliberate change of muscular tension will significantly affect the parameters describing stability. The aim of this study was to investigate the effect of voluntary muscle contraction on postural stability during standing with and without visual feedback in healthy adults.

\section{Material and methods}

Seventeen young adults ( 9 women and 8 men) participated in this study. Their mean age was $24.11 \pm 3.05$ years, body mass $70.76 \pm 16.06 \mathrm{~kg}$ and height $174.82 \pm 8.63 \mathrm{~cm}$. No participants declared any sensory impairment, physical injury, or increased physical or mental stress that hindered the performance of the balance task. In addition, all participants reported practicing physical activity, at least twice per week. Before the measurement, the participants were informed about the procedures used and the possibility of withdrawing from the experiment at any moment. The study protocol was conducted according to the ethical principles of the Declaration of Helsinki and was approved by the institution's ethics committee (SEK 01-09/2020).

Center of pressure $(\mathrm{CoP})$ trajectories were measured using the AMTI AccuSway force platform (Advanced Mechanical Technology Inc., USA) at a sampling rate of $100 \mathrm{~Hz}$. Study participants underwent four trials in the following order: standing barefoot on both feet with eyes open (eo) and with eyes closed (ec). Another two attempts (eot, ect) involved standing in the previous visual conditions with controlled muscle contraction. Each trial took 30 seconds with a 5 minutes break. Each test was recorded once for each participant to reduce the potential effects of learning and fatigue. The last two attempts were aimed at creating a situation in which the subject had to tense the core muscles as 
Tab. 1. The mean and standard deviation of analysed parameters

\begin{tabular}{lcccc}
\hline Parameter & eo & eot & ec & ect \\
\hline XMax $(\mathrm{cm})^{* \#}$ & $0.45 \pm 0.18$ & $0.68 \pm 0.37$ & $0.54 \pm 0.29$ & $0.71 \pm 0.21$ \\
YMax $(\mathrm{cm})^{* \wedge}$ & $1.09 \pm 0.55$ & $1.18 \pm 0.35$ & $1.53 \pm 0.48$ & $1.4 \pm 0.26$ \\
Area95 $\left(\mathrm{cm}^{2}\right)^{*}$ & $1.47 \pm 1.34$ & $1.87 \pm 0.84$ & $2 \pm 1.76$ & $2.59 \pm 1.11$ \\
XMin $(\mathrm{cm})^{*}$ & $-0.46 \pm 0.18$ & $-0.7 \pm 0.32$ & $-0.57 \pm 0.29$ & $-0.73 \pm 0.28$ \\
YMin $(\mathrm{cm}) *$ & $-1.06 \pm 0.45$ & $-1.19 \pm 0.52$ & $-1.42 \pm 0.6$ & $-1.59 \pm 0.4$ \\
Path Length $(\mathrm{cm})$ & $142.89 \pm 53.95$ & $134.64 \pm 56.29$ & $147.43 \pm 77.74$ & $136.26 \pm 52.33$ \\
VAvg $(\mathrm{cm} / \mathrm{s})$ & $4.76 \pm 1.8$ & $4.49 \pm 1.88$ & $4.91 \pm 2.59$ & $4.54 \pm 1.74$ \\
\hline
\end{tabular}

* - statistically significant differences between the trials eo and ect, \#-statistically significant differences between the trials ec and ect,

$\wedge$ - statistically significant differences between the trials eo and ec, $\mathrm{p}<0.05$.

much as possible without articular movement. Involved muscle groups contained: quadriceps femoris, gluteus and abdominal muscles. Additionally, subjects were asked to pull shoulder blades down means retraction of shoulders and the hands were extended. During visual feedback trials, the subjects looked at a point located on the wall at a distance of $1.5 \mathrm{~m}$ from the measuring station, which was in their line of sight. From the Balance Clinic program, which supports the AMTI platform, it is possible to export 35 parameters. The twelve parameters that are the most often analysed in this type of works were selected for the analysis. These included: (1) maximum and minimum displacement from the average of the data in anterior-posterior (Y) and mediolateral (X) direction (X, YMax; X, YMin); (2) the CoP path length for the duration of the trial (PathLength); (3) the average velocity (VAvg); (4) the area of the 95th percentile ellipse (Area95).

\section{Statistical analysis}

Statistical analyses were performed using PQStat v.1.8.2 (PQStat Software, Poznań, Poland), with the significant $\mathrm{p}$-value set at 0.05 . All coefficients were tested for normal distribution, using the Shapiro-Wilk test. Next, the Friedman test is a nonparametric version of repeated measures ANOVA and post-hoc Dunn test with Bonferroni correction was applied to examine differences between the trials for all coefficients.

\section{Results}

Most of the analysed parameters had different distributions than normal. The Friedman test results showed the existence of statistically significant differences between the analysed trials for the following parameters: (1) XMax: F (3, N = 68) = 14.01; p =0.0028; (2) XMin: $\mathrm{F}(3, \mathrm{~N}=68)=15.42$; $\mathrm{p}=0.0014$; (3) YMax:
$\mathrm{F}(3, \mathrm{~N}=68)=15.56 ; \mathrm{p}=0.0013$; (4) YMin: $\mathrm{F}(3, \mathrm{~N}=68)$ $=17.40 ; \mathrm{p}=0.0005 ;(5)$ Area95: $\mathrm{F}(3, \mathrm{~N}=68)=16.17$; $\mathrm{p}=0.0011$. After conducting the post-hoc Dunn test with Bonferroni correction, it was shown that significantly lower values of following parameters: XMax $(p=0.0033)$, YMax $(p=0.00585)$, Area95 $(p=0.0005)$ were recorded for trials with open eyes (eo) compared to those generated during trials with eyes closed and with increased muscle contraction (ect) (Tab. 1). However, for two parameters (XMax and YMax), additional significant differences were noted. Significantly lower values of XMax $(p=0.0316)$ were recorded for the ec trial compared to those obtained during ect. For YMax significantly ( $p=0.0053$ ) lower values were recorded for the eo test compared to those obtained during the ec. For the parameters, XMin $(\mathrm{p}=0.0316)$ and YMin $(p=0.0053)$ significantly higher values were recorded for the test eo compared to those obtained during the test ect (Tab. 1).

The Friedman test results showed exhibited no statistically significant differences between trials for Path Length: $\mathrm{F}(3, \mathrm{~N}=68)=0.45, \mathrm{p}=0.9278$ and for average CoP velocity: $F(3, N=68)=0.45 ; p=0.9278$. The highest mean value of both parameters was recorded for ec and the lowest for eot trials (Tab. 1). It is worth noting that for both parameters, the appearance of increased muscle contraction resulted in a decrease in their values.

\section{Discussion}

The aim of this study was to investigate the effect of voluntary muscular contraction on postural stability during standing with and without visual feedback in healthy adults. It was proved that muscular contraction can negatively affect the standing posture mainly in combination with a lack of visual feedback. This statement 
was confirmed by significantly higher values of maximum and minimum displacement from the average of the data in anterior-posterior (Y) and medio-lateral (X) direction and the value area of the 95th percentile ellipse (Area95) obtained during ect trial compared to these recorded for the trial with eyes open. The same results were obtained by Tassani, Font-Llagunes [24]. Tassani, Font-Llagunes [24] investigated how the increased muscle tension and relaxation effect influence postural control during eyes open and closed conditions. They found that muscle tension significantly reduced the stability of subjects while relaxed states increased stability in absence of sight what suggested that relaxation improves proprioception. Coco, Fiore [7] assessed stress exposure and postural control in young females. They demonstrated a strong correlation between the perceived stress scale (PSS) and total mood disturbance (TMD) scores, as well as the levels of salivary cortisol and the mean value of Area95 measured during standing positions with eyes closed. Moreover, $\mathrm{Coco}, \mathrm{Bu}-$ scemi [8] conducted a similar study on a group of men and they proved that parameter Area95 significantly increased when eyes were closed and the level of stress was high. The same results were obtained in our study. Thus, along with a lack of visual feedback and an increase in muscle contraction, postural stability is reduced.

It is worth emphasizing that reactions in a human posture like freezing and stiffening behaviors with increased muscular tonus are associated with threatrelated situations [31]. Carpenter, Frank [32] proved that humans adopted that type of strategy when they stood under high threat conditions. In this group, the ankle muscle stiffness increased during standing at a height, which was confirmed by increased co-contraction of lower leg muscles. This also caused decreased center of mass (CoM) displacements. Kaminishi, Chiba [33] investigated the effect of tonus on the change in postural control strategy using musculoskeletal simulation. It was demonstrated that decreased muscle tonus has an impact on the difference in strategy shift (ankle or hip). The authors proved that when the posture becomes unstable as the magnitude of tonus decreases a hip strategy. When muscle tonus was large enough the strategy was closer to ankle shift, regardless of muscle weakness. Moreover, Alonso, Van der Elst [34] showed that anterior-posterior sway is related to the ankle strategy, and medio-lateral is related to the hip strategy. Taking into account such mechanisms, it can be seen that during the ect test, the XMin and XMax values were the highest, which would suggest the dominance of the hip strategy associated with increased pelvic girdle muscle tone. However, the highest values of YMax in the ec test and YMin in the ect test show that with the visual control turned off, the ankle muscles assumed the main role of the stabilizers. This theory is confirmed by the stages of body balance regulation presented by Michalak, Przekoracka-Krawczyk [16]. In the case of anterior-posterior direction regulation, there is stage 1 in which modulation of Achilles tendon contraction appears. In the case of medio-lateral regulation (stage 2) change of hip muscle tension is include.

\section{Conclusions}

Healthy and young people who have one control input (vison) taken can maintain a given level of stability. Only the addition of increased muscular activity resulted in a deterioration of stability. Therefore, we can conclude that the ability to relax and contract muscles intentionally is very relevant in controlling balance.

\section{Funding}

This work was supported by the Ministry of Science and Higher Education in the year 2020-2022 under Research Group no 3 at Józef Pilsudski University of Physical Education in Warsaw "Motor system diagnostics in selected dysfunctions as a basis for planning the rehabilitation process".

\section{Conflict of interests}

The authors have no conflict of interest to declare.

\section{References}

1. Horak FB. Postural orientation and equilibrium: what do we need to know about neural control of balance to prevent falls? Age Ageing, 2006; 35 (Suppl 2): ii7-ii11.

2. Kędziorek J, Blazkiewicz M. Nonlinear Measures to Evaluate Upright Postural Stability: A Systematic Review. Entropy (Basel). 2020; 22(12): 1-24.

3. Wang, Z, Hallac R, Conroy CK, White PS, Kane AA, Collinsworth AL et al. Postural orientation and equilibrium processes associated with increased postural sway in autism spectrum disorder (ASD). J Neurodev Disord. 2016; 8: 43.

4. Tjell $\mathrm{CH}$, Halling W, Tenenbaum A. Why do not all recover after a whiplash injury? A hypothesis from an otoneurologic viewpoint. J Whiplash Rel Disorders. 2007; 5(2): 7-23.

5. Knarr BA, Reisman DS, Binder-Macleod S, Higginson JS. Understanding compensatory strategies for muscle weakness during gait by simulating activation deficits seen post-stroke. Gait Posture. 2013; 38(2): 270-5.

6. Kristjansson E, Treleaven J. Sensorimotor function and dizziness in neck pain: implications for assessment 
and management. J Orthop Sports Phys Ther. 2009; 39(5): 364-77.

7. Coco M, Buscemi A, Pennisi E, Cavallari P, Papotto G, Papotto GMF et al. Stress exposure and postural control in young females. Mol Med Rep. 2015; 11(3): 2135-40.

8. Coco M, Buscemi A, Pennisi E, Cavallari P, Papotto G, Papotto GMF et al.Postural Control and Stress Exposure in Young Men: Changes in Cortisol Awakening Response and Blood Lactate. Int J Environ Res Public Health. 2020; 17(19): 1-11.

9. Beelen PE, Kingma I, Nolte PA, van Dieën JH. The effect of foot type, body length and mass on postural stability. Gait Posture. 2020; 81: 241-246.

10. Rossi-Izquierdo M, Santos-Pérez S, Faraldo-García A, Vaamonde-Sáncheaz-Andrade I, Gayoso-Diz P, DelRío-Valeiras M. Impact of obesity in elderly patients with postural instability. Aging Clin Exp Res. 2016; 28(3): 423-8.

11. Paillard T, Noé F. Techniques and Methods for Testing the Postural Function in Healthy and Pathological Subjects. Biomed Res Int. 2015; 1-15.

12. Casabona A, Leonardi G, Aimola E, La Grua G, Polizzi CM, Cioni M et al. Specificity of foot configuration during bipedal stance in ballet dancers. Gait Posture. 2016; 46: 91-7.

13. Imaizumi S, Asai T, Hiromitsu K, Imamizu H. Voluntarily controlled but not merely observed visual feedback affects postural sway. PeerJ. 2018; 6: 1-22.

14. Raffalt PC, Spedden ME, Geertsen SS. Dynamics of postural control during bilateral stance - Effect of support area, visual input and age. Hum Mov Sci. 2019; 67: $1-10$.

15. Blenkinsop GM, Pain MTG, Hiley MJ. Balance control strategies during perturbed and unperturbed balance in standing and handstand. R Soc Open Sci. 2017; 4(7): 1-12.

16. Michalak KP, Przekoracka-Krawczyk A, Naskręcki R Parameters of the crossing points between center of pressure and center of mass signals are potential markers of postural control efficiency. PLoS One. 2019; 14(7): 1-22.

17. Szafraniec R, Barańska J, Kuczyński M Acute effects of core stability exercises on balance control. Acta Bioeng Biomech. 2018; 20(4): 145-51.

18. Ghandali E, Moghadam ST, Hadian MR, Olyaei G, Jalaie S, Sajjadi E. The effect of Tai Chi exercises on postural stability and control in older patients with knee osteoarthritis. J Bodyw Mov Ther. 2017; 21(3): 594-98.

19. Casonatto J, Yamacita CM. Pilates exercise and postural balance in older adults: A systematic review and meta-analysis of randomized controlled trials. Complement Ther Med. 2020; 48: 1-7.

20. Ivanenko Y, Gurfinkel VS. Human Postural Control. Front Neurosci. 2018; 12: 1-9.

21. Caneiro JP, O’Sullivan P, Burnett A, Barach A, O’Neil D, Tveit $\mathrm{O}$ et al. The influence of different sitting postures on head/neck posture and muscle activity. Man Ther. 2010; 15(1): 54-60.

22. Gurfinkel V, Cacciatore TW, Cordo P, Horak FB, Nutt J, Skoss R. Postural muscle tone in the body axis of healthy humans. J Neurophysiol. 2006; 96(5): 2678-87.

23. Benjuya N, Melzer I, Kapłański J. Aging-induced shifts from a reliance on sensory input to muscle cocontraction during balanced standing. J Gerontol A Biol Sci Med Sci. 2004; 59(2): 166-71.

24. Tassani S, Font-Llagunes JM, Ballester MAG, Noailly J. Muscular tension significantly affects stability in standing posture. Gait Posture. 2019; 68: 220-226.

25. Cenciarini $M$ et al. Stiffness and damping in postural control increase with age. IEEE Trans Biomed Eng. 2010; 57(2): 267-75.

26. Maktouf W, Boyas S, Beaune B, Durand S. Differences in lower extremity muscular coactivation during postural control between healthy and obese adults. Gait Posture. 2020; 81: 197-204.

27. Horak FB, Frank J, Nutt J. Effects of dopamine on postural control in parkinsonian subjects: scaling, set, and tone. J Neurophysiol, 1996; 75(6): 2380-96.

28. McFarlane AC. Stress-related musculoskeletal pain. Best Pract Res Clin Rheumatol. 2007; 21(3): 549-65.

29. Feldman R, Schreiber S, Pick CG, Been E. Gait, balance and posture in major mental illnesses: depression, anxiety and schizophrenia. Austin Medical Sciences. 2020; 5(1): 1-6.

30. Stins JF, Ledebt A, Emck C, van Dokkum EH, Beek PJ. Patterns of postural sway in high anxious children. Behav Brain Funct. 2009; 5(1): 42.

31. Adkin AL, Carpenter MG. New Insights on Emotional Contributions to Human Postural Control. Front Neurol. 2018; 9(789): 1-8.

32. Carpenter MG, Frank JS, Silcher CP, Peysar GW. The influence of postural threat on the control of upright stance. Exp Brain Res. 2001; 138(2): 210-8.

33. Kaminishi K, Chiba R, Takakusaki K, Ota J. Investigation of the effect of tonus on the change in postural control strategy using musculoskeletal simulation. Gait Posture. 2020; 76: 298-304.

34. Alonso A, Van der Elst W, Molenberghs G, Buyse M, Burzykowski T.On the relationship between the causal -inference and meta-analytic paradigms for the validation of surrogate endpoints. Biometrics. 2015; 71(1): 15-24. 